\title{
Síndrome de vena cava superior: presentación de seis casos
}

\author{
B. DE LAS HERAS GARCÍA, AM. JIMÉNEZ GORDO, E. CASADO SAENZ, \\ P. ZAMORA AUÑÓN, M. CANTALEJO MOREIRA*, M. GONZÁLEZ BARÓN \\ Servicio de Oncología Médica y *Reumatología. Hospital Universitario La Paz. \\ Universidad Autónoma de Madrid
}

SUPERIOR VENA CAVA SYNDROME: PRESENTATION OF SIX CASES

\section{RESUMEN}

El síndrome de vena cava superior (SVCS), es el conjunto de síntomas y signos derivados de la obstrucción parcial o completa de la vena cava superior. Su diagnóstico precoz, es fundamental para un tratamiento eficaz. Las enfermedades malignas son la causa principal, siendo la más frecuente el cáncer de pulmón. La clínica se caracteriza por la disnea y la triada clásica de edema en esclavina, cianosis facial y circulación colateral tóraco-braquial. En el diagnóstico se utilizan técnicas de imagen, siendo preciso para su confirmación, un diagnóstico histológico. El tratamiento ha de ser lo más precoz posible.

En el presente trabajo se describen los casos de seis pacientes con SVCS, que ingresaron desde el Servicio de Urgencias de nuestro hospital.

Se repasan los principales síntomas y signos, los métodos diagnósticos y el tratamiento empleado, haciendo especial mención a la precocidad en su detención.

PALABRAS CLAVE: Síndrome de vena cava superior. Clínica. Diagnóstico precoz. Tratamiento.

\begin{abstract}
Superior vena cava syndrome is a devastating complication of obs tructive lesions compromising the superior vena cava an its branches. Most cases today are caused by malignant tumors. The most frecuent are lung carcinoma. The clinical presentation is dyspnea, yugular enlarge ment, collateral circulation. Imaging techniques and histologycal confir mation are used for diagnosis. Early diagnosis and treatment is needed.

The following report deals with six patients with superior vena cava syndrome. All entred our hospital through Emergency Deparment.

The description of the principal symptoms and sings, the early diag nosis and the treatment.
\end{abstract}

KEY WORDS: Superior vena cava syndrome. Clinical. Early diagnos tic. Treatment.

De las Heras García B, Jiménez Gordo AM, Casado Saenz E, Zamora Auñón P, Cantalejo Moreira M, González Barón M. Síndrome de vena cava superior: presentación de seis casos. An Med Interna (Madrid) 2001; 18: 369-372.

\section{INTRODUCCIÓN}

El síndrome de vena cava superior (SVCS), es el conjunto de síntomas y signos derivados de la obstrucción parcial o completa del flujo sanguíneo a través de la vena cava superior hacia la aurícula derecha. Puede ser debido a trombosis intrínseca (idiopática o primaria) o a la compresión extrínseca (asociada o no a trombosis secundaria). Constituye una de las escasas situaciones de urgencia médica en Oncología, por lo que su diagnóstico precoz es clave para un eficaz tratamiento y un mejor pronóstico a medio plazo.
En nuestro medio las enfermedades malignas son la causa más habitual del SVCS. Los tumores más frecuentes son los cánceres de pulmón, que suponen el $70 \%$ de todos los casos de SVCS, sobre todo los microcíticos y los linfomas. Existen algunas causas benignas de SVCS que son bastantes raras.

El síntoma más frecuente y precoz es la DISNEA. Los pacientes también pueden referir tos, expectoración hemoptóica, dolor torácico, mareos y trastornos de la visión. La exploración clínica se caracteriza por la triada clásica de: ede ma en esclavina (cara, cuello y ambas regiones supraclaviculares, en ocasiones, si el SVCS está muy evolucionado puede 
verse también edema en miembro superior derecho), cianosis en cara y extremidades superiores y circulación colateral tóraco-braquial. Es habitual, el hallazgo de ingurgitación de las venas yugulares.

Para el diagnóstico se utilizan técnicas de imagen tales como: radiografía de tórax, escaner torácico (TAC), en ocasiones, resonancia magnética y flebografía axilar bilateral. El diagnóstico histológico es fundamental para recomendar un tratamiento específico.

El tratamiento ha de establecerse lo antes posible y se basará en dos aspectos fundamentales: alivio sintomático inicial y tratamiento del proceso maligno primario.

El diagnóstico de sospecha es de extrema importancia, ya que de ello va a depender el pronóstico a largo plazo del paciente cuando la enfermedad neoplásica debuta como un SVCS.

En este artículo exponemos 6 casos clínicos de SVCS vistos en el servicio de Oncología del Hospital La Paz durante los últimos tres meses de 1997. Es importante mencionar que todos ellos ingresaron desde el Servicio de Urgencias, por lo que nos hemos centrado principalmente en los aspectos más relevantes desde el punto de vista de los síntomas y signos clínicos que presentaban los enfermos cuando acudieron al hospital.

\section{CASOS APORTADOS}

En la tabla I se muestran las características de los seis pacientes con SVCS. Todas fueron debidos a cáncer de pulmón, dos casos eran carcinomas microcíticos, otros dos adenocarcinomas y el resto carcinomas epidermoides.

\section{DISCUSIÓN}

Como expusimos en la introducción, la causa más frecuente de SVCS son tumores malignos (75\%-85\%) y dentro de estos el carcinoma de pulmón (1). Dependiendo de las series, la histología más frecuente es el carcinoma de células pequeñas (CMP) $(1,2)$, en otras la distribución es similar entre este y el carcinoma no microcítico (CNMP) (3). En nuestra corta serie predomina el CNMP. Otras causas son los linfomas no Hodking (LNH) y más raramente otros tumores como timomas, tumores de células germinales, tumores metastásicos o enfermedades benignas tales como bocio tiroideo, fibrosis mediastínica, marcapasos, antiguamente, los aneurismas sifilíticos y la mediastinitis tuberculosa. Actualmente una causa en alza, es la yatrogénica debido al uso cada vez más, de catéteres centrales endovenosos para la administración de medicaciones como la quimioterapia. En la infancia la causa más frecuente es la yatrogénica (4).

La clínica se debe a la disminución del retorno venoso en el territorio de la vena cava superior, produciendo un aumento retrógado de la presión venosa en la cabeza, cuello y extremidades superiores, dando lugar a la triada clásica ya expuesta. En nuestros pacientes, el edema en esclavina, la disnea y la ingurgitación de las venas yugulares fueron los síntomas y los signos fundamentalmente observados $(1,4)$, siendo el edema y la disnea los motivos de consulta en urgencias en los seis casos revisados. Otros síntomas que se presentaron con menor frecuencia fueron: somnolencia, cefalea, acúfenos, sensación vertiginosa, dolor torácico, tos, lagrimeo, etc.

TABLA I

CARACTERÍSTICAS CLÍNICAS DE LOS PACIENTES CO N SVCS

\begin{tabular}{|c|c|c|c|c|c|c|}
\hline & Caso 1 & Caso 2 & Caso 3 & Caso 4 & Caso 5 & Caso 6 \\
\hline Edad & 31 & 62 & 74 & 72 & 82 & 46 \\
\hline Sexo & Varón & Varón & Varón & Varón & Varón & Varón \\
\hline Tumor & CMP & CM P & $\begin{array}{l}\text { CNM P } \\
\text { epidermoide }\end{array}$ & $\begin{array}{l}\text { CNM P } \\
\text { epidermoide }\end{array}$ & $\begin{array}{l}\text { CNM P } \\
\text { adenoca }\end{array}$ & $\begin{array}{l}\text { CNM P } \\
\text { adenoca }\end{array}$ \\
\hline Dgto. & Rx.Tórax & Rx.Tórax & Rx.Tórax & Rx.Tórax & Rx.Tórax & Rx.Tórax \\
\hline Sintomas. & Disnea & Disnea & Disnea & Disnea, Cefalea & Disnea & Disnea \\
\hline Signos & $\begin{array}{l}\text { Edema } \\
\text { esclavina }\end{array}$ & $\begin{array}{l}\text { Edema de } \\
\text { M SD }\end{array}$ & $\begin{array}{l}\text { Edema de } \\
\text { MSD, IY }\end{array}$ & $\begin{array}{l}\text { Edema } \\
\text { esclavina, IY }\end{array}$ & $\begin{array}{l}\text { Edema } \\
\text { M SD, CC }\end{array}$ & $\begin{array}{l}\text { Edema } \\
\text { esclavina }\end{array}$ \\
\hline D.SVCS & 8 meses & Debut & 1 año & 2 años & 5 meses & Debut \\
\hline Tratamiento & Q M T & QMT & --- & QM T. & ---- & QM T \\
\hline T.SVCS & RT & Prótesis & RT & RT & Sintomático & Sintomático \\
\hline Evolución & $\begin{array}{l}\text { Exitus } 1 \mathrm{~m} \\
\mathrm{p} \text {-SVCS }\end{array}$ & Vivo con & $\begin{array}{l}\text { Exitus } 1 \mathrm{~m} \text {. } \\
\mathrm{p} \text {-SVCS }\end{array}$ & $\begin{array}{l}\text { Exitus } 1 \mathrm{mp}- \\
\text { SVCS }\end{array}$ & $\begin{array}{l}\text { Exitus } 5 \text { m } \\
\text { p-SVCS }\end{array}$ & $\begin{array}{l}\text { Exitus } 6 \text { m } \\
\text { p-SVCS }\end{array}$ \\
\hline SG & 9 meses & 2,5 años & laño y 1 mes & 2 años y 1 mes & 10 meses & 6 meses \\
\hline
\end{tabular}

CM P: carcinoma microcítico de pulmón, CNM P: carcinoma no microcítico de pulmón. M SD: miembro superior derecho, IY: ingurgitación yugular, CC: circulación colateral, D.SVCS: momento del diagnóstico del SVCS en relación con el primario, Q T: quimioterapia, RT: radioterapia, T. SVCS: tratamiento del SVCS, Adenoca: adenocarcinoma, m: mes, Vivo con: vivo con enfermedad, p-SVCS: después del diagnóstico del SVCS, SG: supervivencia global. 
Para llegar al diagnóstico de SVCS se pueden utilizar diversas técnicas de imagen (4).

La más importante de ellas es la radiografía de tórax. Es la técnica de elección y al alcance de cualquiera, en un paciente que acude a urgencias y en el que sospechamos un SVCS. Generalmente muestra una masa, localizada en hemitórax derecho, en aproximadamente $90 \%$ de los casos, siendo el hallazgo más frecuente el ensanchamiento mediastínico (5) (Fig. 1), en ocasiones también se observa derrame pleural. El TAC torácico nos da una información más detallada sobre la masa pulmonar, y la afectación ganglionar, así como de las estructuras vecinas afectas y de la posible trombosis de la vena cava superior asociada.

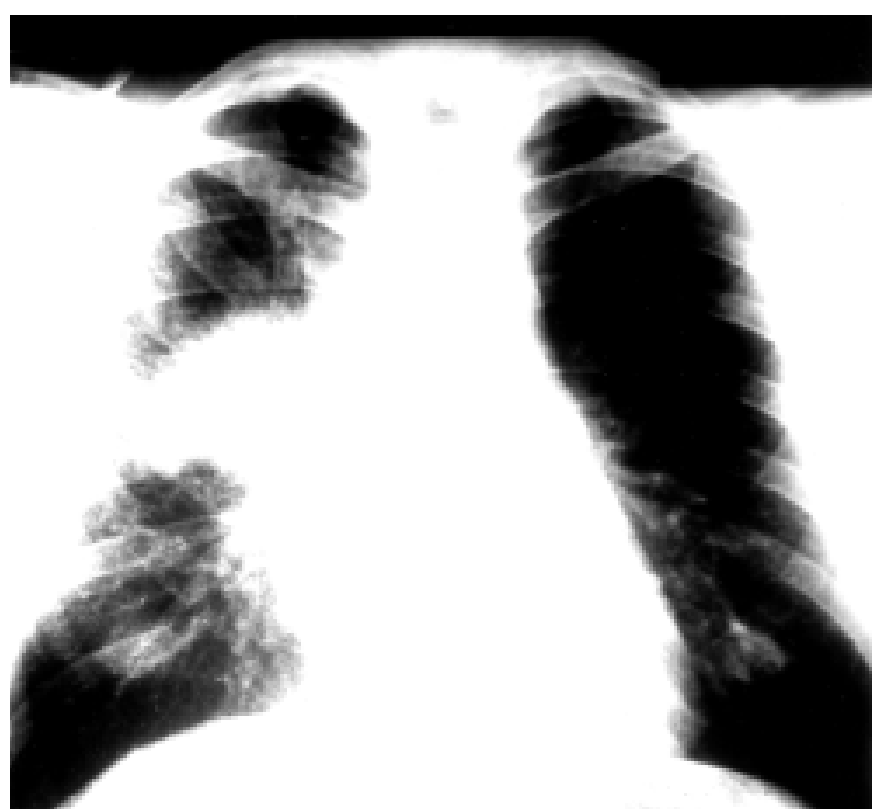

Fig. 1. Radiografía de tórax que muestra un ensanchamiento mediastínico.

La RNM puede también emplearse, aunque su papel no está bien definido todavía.

La flebografía axilar bilateral (6) nos da información de la extensión de la obstrucción y del trombo asociado, lo que puede ser útil en los raros casos en que se plantee la cirugia de bypass. Ahora bien no todos los autores recomiendan su utilización y generalmente se usa sólo en los SVCS de causa benigna.

Por último, es fundamental un diagnóstico histológico, ya que en casos de SVCS de etiología maligna, la evolución y pronóstico dependen, sobre todo, de la agresividad del tumor. Por otra parte, solo un correcto diagnóstico de naturaleza nos va a permitir recomendar un tratamiento específico. Se suele realizar mediante punción aspiración con aguja fina de la masa torácica bajo control radiológico o bien mediante fibrobroncoscopia en caso de ser una lesión broncopulmonar.

La radiografía de tórax, fue la primera técnica utilizada en el diagnóstico de nuestros pacientes y nos permitió en todos los casos confirmar el diagnóstico clínico de sospecha. El TAC y la confirmación históloga del tumor primario se realizaron, pero en un segundo tiempo, cuando el enfermo estaba ingresado.
El tratamiento del SVCS se basa en dos aspectos fundamentales: alivio de los síntomas y tratamiento específico de la causa. El primer punto es la base de actuación del médico en la urgencia hospitalaria. Se suele recomendar reposo en cama, oxigenoterapia para aliviar la disnea, dieta pobre en sal, diuréticos tipo furosemida, (aunque estos están cada vez más discutidos), para reducir el edema y corticoides a dosis altas para reducir la inflamación y aliviar la congestión y el edema. En los casos de SVCS secundario a trombosis por catéteres se pueden emplear trombolíticos tipo uroquinasa o estreptoquinasa (4).

Dentro de las medidas paliativas de reciente introducción en el tratamiento del SVCS está el empleo de Stents, prótesis de metal autoexpandibles. Se colocan con anestesia local y bajo control radiológico, y han permitido el seguimiento de algunos pacientes entre 2-17 meses, con una media de 6 meses (7-9) (Fig. 2). Su funcionalidad se valora mediante la utilización del ECO-doppler de la vena mamaria interna, que nos da una visión exacta del flujo sanguíneo a su través (10). Se han obtenido unos resultados excelentes en el alivio rápido de síntomas, tales como cefalea, ingurgitación yugular y circulación colateral, ya en las 24 horas posteriores a su colocación, excepto para el edema y la disnea, síntomas que son algo más resistentes. Además el uso de Stents, no es una contraindicación para un tratamiento específico posterior (quimioradioterápico) (10). La única precaución que requieren, para evitar la retrombosis, es el tratamiento profiláctico durante algunas semanas con heparinas de bajo peso molecular. Nosotros sólo hemos utilizado este procedimiento en un paciente, obteniendo un buen resultado clínico, con una media de seguimiento hasta la fecha de cinco meses.

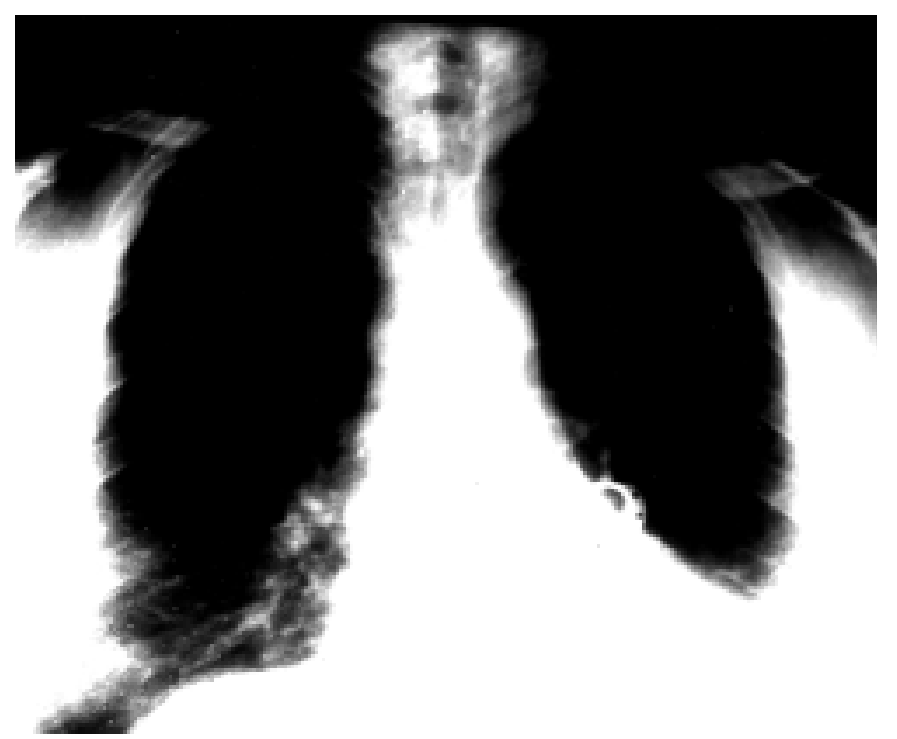

Fig. 2. Stent colocado en la cava superior.

En lo que se refiere al tratamiento del proceso maligno primario, suele ser en la mayoría de los casos la quimioterapia. La combinación con radioterapia se emplea sobre todo en los cánceres de pulmón, especialmente los microcíticos (11) y también como consolidación tras la quimioterapia en los linfomas. El tratamiento físico puede conseguir alivio sintomático en más del $80 \%$ de los pacientes (2). 
Los pacientes que debutan con un SVCS como manifestación inicial de un tumor maligno, tienen un peor pronóstico en comparación con estos que lo desarrollaron después del diagnóstico inicial. La supervivencia es mejor en los pacientes con linfomas, seguido por el timoma maligno y CMP, que aquellos con CNMP y tumores metastásicos (12).

Para concluir, podemos decir, que el diagnóstico del SVCS es fundamentalmente clínico, de ahí la importancia que

\section{Bibliografía}

1. Hinojosa MC, Fernández I, Ergueta P, González E. Prevalencia Etiológia del síndrome de vena cava superior. An Med Interna (Madrid) 1998; 15: 531-533.

2. Hoegler D. Radiotherapy for palliation of symptoms in incurable cancer. Curr Probl Cancer 1997; 21: 129-83.

3. Egelmeers A, Goor C, Van Meerbeeck J, Van den Weyngaert D, Scalliet P. Palliative effectiveness of radiation therapy in the treatment of superior vena cava syndrome. Bull Cancer Radiother 1996; 83: 153-7.

4. Arroyo Yustos M, León Carbonero AI, De Castro Carpeño J. Síndrome de vena cava superior. En "Tratado de Medicina Paliativa y tratamiento de soporte en el enfermo con cancer”. Eds. Gónzalez Barón M. Primera edición. Editorial Panamericana 1996; 675-84.

5. Laguna P, Gazapo T, Murillas J, Martín H, Portero JL, Moya M. Síndrome de vena cava superior: análisis de una serie de 81 casos. An Med Interna (Madrid) 1998; 15: 470-475. tiene la sospecha en el servicio de urgencias, que se podrá confirmar con una exploración complementaria tan simple como una radiografía de tórax. La detección precoz de la "triada clásica" ya mencionada, junto con el síntoma principal DISNEA, en pacientes portadores o no de patología tumoral, es fundamental para iniciar el tratamiento específico, que redundará en una mejor calidad de vida y más larga supervivencia.

6. Mahmud AM, Isawa T, Teshima T, Hirano T, Anazawa Y, Miki M, Nukiwa T. Radionuclide venography and its functional analysis in superior vena cava syndrome. J Nucl Med 1996; 37: 1460-4.

7. Shah R., Sabanathan S., Lowe RA, Mearns AJ. J Thorac Cardiovasc Surg 1996; 112: 335-40.

8. Gross CM, Kramer J, Waigand J, Uhlich F, Schroder G, Thalhammer C, Dechend R, Gulba DC, Dietz R. Stent implantation in patients with superior vena cava syndrome. Am J Roentgenol 1997; 169: 429-32.

9. Chacón JI, García L, Lanciego C, Mora MA, Peris A, Martinez B, López L. Am J Clin Oncol 1997; 20: 293-297.

10. Martinoli C, Cittadini G, Gandolfo N, Crespi G, De Caro G, Derchi LE. Radiology 1997; 204: 865-70.

11. Chan RH, Dar AR, Yu E, Stitt LW, Whiston F, Truong P, Vicent MD, Kocha WI. Int J Radiat Oncol Biol Phys 1997; 38: 513-20.

12. Chen YM, Yang S, Perng RP, Tsai CM. J Clin Oncol 1995; 25: 32-6. 